\title{
Ciencias naturales en primaria en los años setenta en México: ¿Una reforma entre revoluciones?
}

\author{
Elementary science in the seventies in Mexico: \\ A reform between revolutions?
}

\section{María del Mar Estrada Rebull ${ }^{1}$}

\begin{abstract}
Resumen
En la década de 1970, en el marco de la reforma educativa de Luis Echeverría, un grupo liderado por científicas y científicos mexicanos diseñó una gran reforma curricular de ciencias naturales en primaria. Se ha explicado que esta reforma fue una réplica con retraso de la llamada "primera revolución" en educación en ciencia, originada en Estados Unidos, que buscaba una enseñanza de las disciplinas científicas "tal cual eran" mediante el método científico y la experimentación. Este artículo va más allá de esa explicación: analiza el contexto de la reforma, su proceso de diseño y sus libros de texto bajo una mirada histórica, filosófica y social sobre la ciencia y la tecnología. Muestra cómo la reforma abrevó de largas tradiciones del mundo occidental, y a la vez reflejó significados locales de la naturaleza, el campo, la ciencia, la tecnología y su papel para la niñez y el México de entonces.
\end{abstract}

Palabras clave: historia de la educación, educación en ciencia, ciencia, tecnología, sociedad

\section{Abstract}

In the 1970s, a group led by Mexican scientists designed an important elementary science curriculum reform, in the context of then president Luis Echeverría's larger education reform. This curriculum reform has been explained as a delayed replica of the so-called "first revolution" in science education that originated in the United States, which promoted teaching scientific disciplines "as they were", through the scientific method and experimentation. This article goes beyond such explanation by

\footnotetext{
${ }^{1}$ Universidad de Michigan - Escuela de Educación. Correo electrónico: marestra@umich.edu
} 
analyzing the reform's context, design process, and textbooks from a historical, philosophical and social lens on science and technology. It shows how the reform drew from long-standing western traditions, while also expressing local meanings about nature, agriculture, science, technology, and their role for children and society in 1970s Mexico.

Keywords: history of education, science education, science, technology, society

\section{Introducción}

La ciencia moderna tomó algo de la autoridad que antes tenían las religiones. La ciencia parecía ofrecer respuestas plausibles a ciertas preguntas cósmicas, aunque no ayudaba mucho con las cuestiones morales. La ciencia adquirió aun mayor prestigio conforme se fue imbricando con la innovación tecnológica y los avances médicos. Casi todos los sistemas escolares en el mundo incluyeron sus métodos y sus ideas entre aquello que los niños y niñas habian de aprender.

McNeilly McNeill, 2003: 279.

En su libro sobre la historia de la humanidad "a vista de pájaro", McNeill y McNeill argumentan que las sociedades se conformaron en redes cada vez más grandes y complejas, que llegaron a ser globales mucho más pronto de lo que imaginamos. La red global contaba ya cientos de años cuando en el siglo XIX llegó la ciencia a la educación básica de los estados modernos. Esta llegada fue parte de las transformaciones culturales de gran calado en la historia reciente de la humanidad. La rápida expansión de la cobertura escolar en los siglos XX y XXI ha hecho que, al día de hoy, la gran mayoría de las personas en el mundo hayamos participado en la ciencia escolar, en alguna de sus versiones, a través de la educación primaria. $\mathrm{Ha}$ habido numerosos debates sobre cuáles son exactamente los métodos e ideas de la ciencia "que los niños y las niñas han de aprender", como dicen McNeill y McNeill (2003: 279). En estos debates también se han jugado los múltiples significados de la ciencia y la tecnología en sucesivos momentos culturales, políticos y económicos. Bajo esta mirada -la de los estudios históricos, filosóficos y sociales sobre ciencia y tecnología-, revisaremos un importante episodio mexicano: la reforma del currículo de ciencias naturales en primaria en los años setenta, que se mantuvo vigente por dos décadas, y cuyos libros acompañaron millones de infancias en nuestro país.

Cuando se trata de la ciencia y la tecnología en países del Sur global como México, muchas veces se piensa que estos países son la "periferia", a donde llega con retraso y con distorsiones la verdadera ciencia y tecnología producida en el "centro"; es decir, en los países más occidentales, ricos e industrializados. En cambio, historiadoras, filósofos y sociólogas de 
la ciencia de los países del Sur han mostrado que la ciencia y la tecnología del Sur tienen sus propias lógicas y sus propias participaciones en la ciencia y la tecnología globales (Gorbach y López, 2008). También la reforma curricular de ciencias naturales en los setenta se ha explicado en términos de centros y periferias: se ha dicho que esta reforma fue producto de una influencia con retraso respecto de la "primera revolución" estadounidense en la enseñanza de las ciencias naturales. (ver León et al., 1995: 35). Pero esta explicación no resulta suficiente: adoptaremos la mirada de los estudios históricos, filosóficos y sociales sobre ciencia y tecnología desde el Sur parar restaurar los significados propios de la reforma mexicana de los setenta.

Para situar el análisis, revisaremos algunos hitos y tradiciones de pensamiento sobre educación en ciencia a nivel internacional, incluyendo la primera y a la segunda "revoluciones" y su periodo intermedio, donde se sitúa la reforma mexicana de los setenta. Después plantearemos el contexto político en que se dio dicha reforma: el sexenio de Luis Echeverría, sus esfuerzos por legitimar el régimen priísta tras los hechos del 68, su retórica de izquierda y su política científica, tecnológica y educativa. Consideraremos quiénes recibieron el encargo de reformar el currículo de ciencias naturales -un grupo liderado por científicos y científicas mexicanos adscritos a instituciones de educación superior, que conformaron el DIE-CINVESTAV a partir de este primer proyecto-; desde dónde partieron y cómo lo hicieron. Luego analizaremos el currículo mismo y los libros de texto en que se plasmó. Mostraremos cómo la reforma abrevó de largas tradiciones del mundo occidental, y a la vez reflejó significados locales de la naturaleza, el campo, la ciencia, la tecnología y su papel en el México de entonces. Para realizar estos análisis se tomó en cuenta literatura especializada internacional y nacional (incluyendo escritos de los mismos reformadores). Se revisaron documentos de la época relacionados con la reforma, así como todos los libros para alumnos y para docentes de la misma, privilegiando las primeras ediciones siempre que pudieron encontrarse. También se consultó a Antonia Candela, una de las principales autoras del currículo.

\section{Linajes locales y globales de la educación en ciencia}

Desde sus inicios, la educación en ciencia en México ha sido partícipe de entramados globales y especificidades locales. Desde el virreinato ha existido una tradición de ciencia secular, empirista, experimental y utilitarista, que tuvo también vertientes educativas: "Las corrientes científicas modernas se difundieron a través de los esfuerzos pedagógicos de los jesuitas y más tarde mediante el periodismo ilustrado de los criollos, hasta consolidarse en las cátedras de las modernas instituciones borbónicas" (Azuela, 2010: 173). Después de la independencia, el sistema educativo mexicano nació y fue consolidándose junto con los proyectos de nación de la reforma y el porfiriato. En ese periodo se decretó que la educación 
sería moderna, científica, positivista y comtiana, con asignaturas científicas adaptadas a partir de la consulta de planes de estudio a nivel internacional, y con influencia de los pedagogos reformadores europeos del siglo dieciocho. Así, aunque con antecedentes particulares, el siglo XX mexicano nació con la enseñanza de la ciencia inserta en el currículo de primaria en una tónica similar a la internacional. Las ciencias habían llegado a los planes de estudio para quedarse. En los siguientes periodos educativos en México -el vasconcelista (declaradamente antipositivista) y el socialista, según la periodización de Pablo Latapí (1998: 21-42)-, aunque con distintos énfasis y objetivos, las ciencias experimentales con base empírica siguieron considerándose parte importante de la educación elemental, y desde entonces ha sido así.

Desde finales del siglo XIX e inicios del XX, Estados Unidos tomó liderazgo en las innovaciones: en solo medio siglo se gestaron ahí muchos de los enfoques y debates de la educación en ciencia que hasta ahora tienen vigencia. Por un lado, apareció la corriente de la Ciencia Escolar Elemental, liderada por W.T. Harris y E.G. Howe, quienes formularon los primeros currículos completos de ciencia para el nivel primaria. Novedosamente, éstos se estructuraban en torno a generalizaciones científicas más que a hechos o datos aislados, e incluían el trabajo empírico y de laboratorio como método de aprendizaje. Pero su principal apuesta pedagógica era la lectura de textos elaborados ad hoc, por lo que Alan McCormack denominó este periodo (1920-1957) como el periodo de los libros de texto (McCormack, 1992: 17), siendo los de Gerald Craig los más emblemáticos. Por otro lado, surgió la corriente del Estudio de la Naturaleza, basada en la literatura romántica y en los reformadores educativos europeos. Su énfasis no era la adquisición de conocimiento, sino el desarrollo personal y social (Bybee y DeBoer, 1994: 366), así como la recuperación del interés por la agricultura frente al éxodo de la población hacia las ciudades. De estos dos, el enfoque que terminó predominando e informando las propuestas curriculares de los años cincuenta fue la Ciencia Escolar Elemental, que ponía el centro el aprendizaje de las disciplinas (López y León, 2003: 377).

La segunda mitad del siglo XX trajo nuevos movimientos a nivel internacional. El lanzamiento del satélite ruso Sputnik detonó la "primera revolución" en la enseñanza de las ciencias (término acuñado por McCormack, 1992). En el contexto de la Guerra Fría y la Carrera Espacial, Estados Unidos interpretó el Sputnik como una terrible derrota, y como signo de un rezago generalizado en materia de ciencia, la cual se consideraba crucial para el progreso económico y social. Por entonces emergía el paradigma de la big science, que implicaba la participación coordinada de numerosos científicos e ingenieros de diversas disciplinas y el financiamiento público y privado en un contexto de armamentismo y nacionalismo exacerbados. Así, el Sputnik azuzó también un movimiento sin precedentes para mejorar la enseñanza de la ciencia y la tecnología en los niveles básicos y para expandirla a nivel profesional. 
La primera revolución se caracterizó por que científicos profesionales se organizaron en sociedades y comisiones para actualizar y mejorar los programas (McCormack, 1992: 17-18), con la consigna de "enseñar la ciencia tal como es" (Gutiérrez-Vázquez, 1984: 96). Se reivindicaba una enseñanza "dura" de las disciplinas científicas, dejando de lado objetivos como la comprensión de la naturaleza de la ciencia, su papel en la sociedad, sus aplicaciones tecnológicas cotidianas, o el entrenamiento mental, propio de siglos anteriores. Enseñar las ciencias "tal como son" implicaba romper con el método libresco del periodo anterior y poner por delante el método propio de las ciencias -el experimental- como forma de enseñanza y aprendizaje de las disciplinas. Así, se incluyó por primera vez al laboratorio de forma sistemática y extendida en las escuelas. El proceso de la educación de Jerome Bruner (1960) también resultó una influencia decisiva, al postular que la ciencia cuenta con conceptos fundamentales que le dan coherencia, unidad y organización para su enseñanza gradual a estudiantes de todas las edades (repitiendo los mismos temas a modo de espiral: primero abordándolos en lo particular y simple, luego de forma más general y compleja).

Sin embargo, las flamantes propuestas de la primera revolución solo pudieron implementarse y dar resultados notables en contextos privilegiados, y en un porcentaje pequeño de los casos. Tanto en Estados Unidos como en el Reino Unido, la mayoría de los profesores continuaban dando sus clases como antes, ya que los conocimientos requeridos y los cambios implicados, en general les eran ajenos (McCormack, 1992: 18; Akker, 1998: 426; López y León, 2003: 377-378). Además, vinieron giros constructivistas en la pedagogía y en la investigación educativa, y con ellos, una ola de críticas a las ideas positivistas sobre la ciencia que la primera revolución entrañaba. Así, el paradigma se volvía insostenible por todos los frentes, y se auguraban nuevas corrientes.

Durante la primera revolución no solo se realizaron grandes proyectos en países ricos, sino también en otros lugares del resto del mundo: se gastó más para desarrollar los currículos de ciencias naturales que los de cualquier otra asignatura. En un inicio se adoptaron y tradujeron los ejemplos estadounidenses y británicos, pero después se realizaron adaptaciones. Éstas tomaron en cuenta aprendizajes de la implementación de los originales, por lo que incluyeron ya características de la segunda revolución. Habría entonces una corriente intermedia, que incluiría la reforma mexicana de los setenta. Juan Manuel Gutiérrez-Vázquez fue quien lideró esta reforma, y participó después en muchas otras alrededor del mundo. Gutiérrez-Vázquez consideraba que la corriente intermedia fue influenciada por la idea de enseñar las ciencias mediante el método científico, pero también por los cambios dentro de la ciencia misma: por "el gran éxito de productividad teórica y práctica de las fronteras interdisciplinarias y de los enfoques multi e interdisciplinarios (bioquímica, biofísica, biofisicoquímica, biología molecular, electroneurofisiología, cibernética, etc.)" (Gutiérrez-Vázquez, 2004: 98). Pensaba que ambas influencias tuvieron por resultado emparentar las distintas disciplinas científicas 
entre sí, dando lugar a un enfoque de "ciencia integrada". Ello conllevó grandes retos organizativos para producir ambiciosos materiales que, a su juicio, fracasaron en su mayoría, a diferencia de la reforma mexicana que él encabezó.

Después vendría la llamada segunda ola (Akker, 1998: 427) o segunda revolución (McCormack, 1992) a partir de los años ochenta, o incluso desde los setenta, para otros autores (Wallace y Louden, 1998). Esta no tuvo un detonante definitorio, ni tampoco trajo la gestación de grandes proyectos. Se caracterizó más bien por la proliferación de debates que poco a poco influyeron en desarrollos curriculares (con equipos más interdisciplinarios, donde el rol de los científicos era menos prominente) y de estrategias de formación docente. Dos productos de esta corriente fueron el programa Chemistry in the Community de la American Chemical Society, y el reporte Science for All Americans, que brindaba lineamientos para la alfabetización científica. La segunda revolución se caracterizó también por la importancia que daba al "conocimiento relevante" y a los aspectos históricos y culturales de la ciencia y la tecnología en el currículo (Pedretti y Nazir, 2011); lo que se conoce como la corriente Ciencia, Tecnología y Sociedad (CTS).

La reivindicación de una formación más general, en lugar del énfasis en las disciplinas científicas, no solo provino de las críticas al enfoque anterior, sino también del hecho de que cada vez crecía más la población que ingresaba y permanecía en el sistema educativo, por lo que sus características, necesidades y expectativas se volvieron más heterogéneas. Esto propició el replanteamiento de los objetivos, e invitó a preguntarse por la perspectiva de grupos tradicionalmente subrepresentados en la enseñanza de la ciencia y la tecnología: las niñas y las minorías (Akker, 1998: 428). Surgieron también preguntas sobre cómo la ciencia, producto de una cultura y una cosmovisión masculina y europea, podía resultar en currículos incluyentes y liberadores para todas las culturas (Wallace y Louden, 1998: 477).

Para León et al., tanto la primera como la segunda revolución estadounidenses se dieron también en México, "pero con un retraso considerable" (León et al., 2005: 35). La reforma mexicana de los setenta, entonces, se habría dado por la influencia con retraso de la primera revolución estadounidense. Desde el posterior paradigma constructivista (años ochenta en adelante), la reforma de los setenta también fue agrupada con la primera revolución, y como tal fue etiquetada de positivista o empirista (Núñez, 1980, s/p.). En este artículo procuraremos comprender la reforma en su significado propio, más allá de su ubicación entre dos revoluciones. 


\section{La reforma educativa de Echeverría como marco de la reforma curricular en ciencias}

La decisión de cambiar el currículo de primaria y secundaria fue parte de la gran reforma educativa del régimen de Luis Echeverría (1970-1976), que fue clave en su búsqueda de legitimar su régimen y diferenciarlo del de Gustavo Díaz Ordaz tras la represión a los movimientos de 1968.

En consonancia con el discurso echeverrista de democratización y tolerancia a la disidencia, se conformó una Comisión Plural para la reforma educativa, se realizó una consulta a más de 25 mil personas y se reclutó a ex disidentes en puestos clave. En lo económico, el régimen adoptó el desarrollo compartido (crecimiento económico con reparto equitativo), que en materia educativa se reflejó en una expansión de servicios educativos para grupos populares, rurales e indígenas, así como en nuevos mecanismos para la acreditación de saberes y habilidades laborales (Meneses, 1991; Kobayashi et al., 1976) y en la diversificación de materiales y medios de promoción educativa y cultural (Kobayashi et al, 1976; Corona y Santiago, 2011). Se mejoraron también las condiciones salariales y laborales del magisterio. El régimen echeverrista suscribía la solidaridad internacional y el tercermundismo, lo cual implicaba impulsar la autosuficiencia tecnológica y la atención al campo para aumentar la productividad. Como otros países "subdesarrollados" que buscaban activar políticas científico-tecnológicas nacionales propias (Leff, 1979), México formuló su primera política en ciencia y tecnología y creó el Consejo Nacional de Ciencia y Tecnología (CONACYT). En ese sexenio se procuró impulsar la ciencia y la técnica, sobre todo en lo agropecuario y pesquero. Aunado a todo ello, había una clara intención de modernizar y expandir los servicios y materiales educativos en general, en respuesta al aumento poblacional y la creciente urbanización (en continuidad con el Plan de 11 años de Jaime Torres Bodet) (Latapí, 1998; Fuentes, 1979). Se crearon los modernos Colegios de Bachilleres y Colegios de Ciencias y Humanidades, así como la Universidad Autónoma Metropolitana y otras universidades. En educación básica, se efectuó la reforma que aquí nos ocupa: se renovaron los planes, programas, libros y los métodos de enseñanza que estos prescribían. Se buscó que el nuevo currículo tuviera una factura académica, científica y contemporánea. Muchos consideraron estos cambios como una revolucionaria ruptura con la tradición escolar (Kobayashi et al., 1976; Latapí, 1998). 


\section{Los y las reformadoras del currículo de primaria}

Roger Díaz de Cossío, entonces subsecretario de Planeación de la Secretaría de Educación Pública (SEP), tuvo la visión de poner a instituciones de educación superior y a científicos y académicos reconocidos al frente de la elaboración de los programas. Para coordinar la reforma en ciencias naturales invitó a Juan Manuel Gutiérrez-Vázquez del Instituto Politécnico Nacional (IPN); para coordinar la de español, a Gloria Bravo Ahúja del Colegio de México (COLMEX); para la de ciencias sociales, a Josefina Vázquez también del COLMEX y para la de matemáticas, a Carlos Imaz del Centro de Investigación y de Estudios Avanzados del Instituto Politécnico Nacional (CINVESTAV). Se trataba de poner el conocimiento de la academia al servicio de la educación básica; en contraste con los libros de texto anteriores, que habían estado a cargo de maestras y maestros normalistas ligados a la SEP. Díaz de Cossío también auspició la idea de que el desarrollo curricular debía ser un proceso de largo plazo, que incluyera la formación de equipos interdisciplinarios cuyo trabajo, lejos de concluir con la entrega de los productos, continuara en el campo de la investigación, desde la cual se pudieran fundamentar las decisiones educativas.

Estos planteamientos convencieron al microbiólogo y ex rector de la Escuela de Ciencias Biológicas del IPN, Juan Manuel Gutiérrez-Vázquez, para participar en este proyecto del gobierno, aun cuando había sufrido persecución policial y un breve exilio tras su actuación en el conflicto del 68 (cuando había impedido la entrada de las fuerzas públicas en el plantel el IPN que dirigía). Otros de los jóvenes científicos reclutados para la reforma en ciencias naturales también mostraron una resistencia inicial (DIE-CINVESTAV, 2014), pero les convenció la promesa de libertad académica, la afinidad con el proyecto echeverrista y la posibilidad de incidencia que representaba elaborar libros de ciencias naturales con un nuevo enfoque educativo e ideológico, que llegarían a manos de todos los alumnos y maestros del país (Candela, 1989: 6). A decir de los reformadores, su autonomía sí fue respetada. Refieren, por ejemplo, que querían poner una foto de una niña indígena descalza en uno de los libros, y el secretario Bravo Ahúja indicó que se excluyera, porque daba una mala imagen de la niñez mexicana. El equipo respondió que, si no se incluía la imagen, todos renunciarían, y el secretario aceptó incluirla (DIE-CINVESTAV, 2014).

Así, al abrigo de la solidez académica, la continuidad institucional y la independencia respecto de la estructura burocrática de la SEP para realizar el proyecto, se fundó el Departamento de Investigaciones Educativas del Centro de Investigación y de Estudios Avanzados del Instituto Politécnico Nacional (DIE-CINVESTAV). Esta institución se encargaría del diseño de los programas y de los libros de texto para los seis grados, con sus respectivos Auxiliares Didácticos para docentes. El DIE-CINVESTAV nació también "con el claro designio de constituir y consolidar a su vez un grupo de investigación" (De Ibarrola, 1989: 56). Por 
entonces casi no había expertos en educación en ciencia en México, ni un corpus empírico o teórico del cual partir. Sin embargo, las recientes teorías de la dependencia planteaban la necesidad de que los países subordinados abandonaran teorías sociales ajenas y desarrollaran las propias. Existía una disposición a la innovación en ciencias sociales, misma que influyó también en el DIE-CINVESTAV (De Ibarrola, 1989).

La estrategia de encargar a científicos la elaboración de programas y materiales de ciencia tenía antecedentes internacionales en la década de los sesenta. Esta tendencia después fue juzgada como una falla, que se intentó remediar conformando equipos más plurales, pero en la práctica solía prevalecer la visión de los científicos (Duschl, 1985; McCormack,1992; Gutiérrez-Vázquez, 2004: 92-103). Gutiérrez-Vázquez defendió que éste no fue el caso de su reforma; no fue la "de los científicos" como se le describió después, sino que su equipo fue realmente interdisciplinario. En él participaron científicos y científicas jóvenes asesoradas por otros ya consagrados, pero también participaron maestras, ilustradores, fotógrafas y diseñadores gráficos (Gutiérrez-Vázquez, 1995: 32). Antonia Candela era entonces una joven física de la Universidad Nacional Autónoma de México (UNAM) que trabajaba con el reconocido físico Luis de la Peña, y pasó a tener un rol preponderante en la reforma de ciencias naturales. Ella describe un estilo de trabajo horizontal y dialógico entre el diverso equipo (Candela, 1989: 8).

La mayor parte de las lecciones de biología las realizó Judith Espinosa con asesoría de Dalila Martínez de Muñoz (CINVESTAV), Sergio Estrada O. y Guillermo Carvajal (Escuela Nacional de Ciencias Biológicas, IPN). El mismo Juan Manuel Gutiérrez-Vázquez elaboró algunas de las lecciones, especialmente las relacionadas con el cuerpo humano y la salud. Otras, en una segunda etapa de la reforma, las elaboró Rosa Vera (Escuela Nacional de Ciencias Biológicas, IPN). Como Candela, Vera también se quedó en las filas del DIE-CINVESTAV. La antropóloga Elsie Rockwell, que trabajaba en la SEP, también se integró a la reforma y al DIE: elaboró algunas lecciones de nutrición. En astronomía, Deborah Dultzin, José Franco y Antonio Lazcano (Instituto de Astronomía, UNAM) fueron los principales autores. Ellen Davis elaboró lecciones de geofísica con el apoyo de Servando de la Cruz y la asesoría de Cinna Lomnitz (Instituto de Geofísica, UNAM), mientras que Horacio García (Facultad de Química, UNAM) elaboró las de química, y Ana María Rocabert (psicóloga de la UNAM), "ayudó al trabajo general y realizó algunas lecciones sobre comportamiento animal y salud mental" (Candela, 1989: 7).

La fotógrafa Mariana Yampolsky también jugó un rol central, porque en la concepción de Gutiérrez-Vázquez, las imágenes en sí mismas -el diseño editorial, las ilustraciones, las fotografías- constituían en buena medida al libro de texto. Así, además de aportar fotografías y coordinar la inclusión de imágenes, el rol de Yampolsky consistió en concebir los libros y las lecciones como un todo visual. 


\section{Influencias y puntos de partida}

María Salud Núñez llegó al DIE-CINVESTAV en los ochenta, tras estudiar en la escuela psicogenética con Jean Piaget y Rolando García en Ginebra. Desde esta perspectiva constructivista consideró que la reforma de los setenta fue "completamente empirista", y criticó que no hubiera tomado en cuenta las etapas de desarrollo de los niños ni sus procesos de construcción del conocimiento científico. En un reporte, señaló:

\footnotetext{
Hasta cierto punto resulta explicable que en el momento en que se abordó en México los cambios curriculares de ciencias naturales, los libros de texto correspondientes se hayan basado en una concepción empirista y, en algunos aspectos en un enfoque psicológico neo-conductista, ya que en esa época y un aun actualmente constituye la corriente predominante en nuestro medio científico y la que ha invadido el campo educativo. Menos explicable y disculpable nos parece el que no se haya tomado en cuenta a los maestros y que no se haya pensado seriamente en el problema de la formación. (Núñez, 1980, s/p.)
}

Los mismos autores y autoras asumieron estas caracterizaciones y críticas. Sin embargo, al momento de elaborar la reforma, el equipo no había partido de compromisos teóricos específicos, en parte porque no existía un campo especializado en educación en ciencia en el país. ¿Cuáles fueron entonces sus puntos de partida?

Como contexto, recordemos que al momento en que el equipo recibió su encargo, se acumulaba un corpus de más de un siglo de pedagogía moderna y progresista (general; no específica de las ciencias naturales), que reivindicaba un aprendizaje activo. Aunque esta tradición distaba de ser el hilo negro, en los setenta se reeditaba como una novedad, en oposición a la tradición (como se había hecho ya en anteriores reformas desde el porfiriato, y como se sigue haciendo hasta la fecha con cada reforma). El discurso pedagógico modernizante estaba permeado en la cultura educativa de la época.

Un documento que así lo sugiere son los Comentarios a los fundamentos del plan de estudios y programas de educación primaria (SEP, 1972c). Su autor, Víctor Hugo Bolaño, era entonces Director General de Mejoramiento Profesional del Magisterio, y fungió como ideólogo y difusor técnico-pedagógico de la reforma. En sus Comentarios, Bolaño recupera y glosa los lineamientos que la Comisión Coordinadora de la Reforma Educativa emitió tras tomar en cuenta los resultados de la amplia consulta pública que se realizó al respecto, por lo que resulta un documento revelador sobre las ideas pedagógicas y sobre ciencia y educación que circulaban, y de las cuales nuestras reformadoras y reformadores de ciencia probablemente participaban. En el documento, Bolaño habla de una enorme expectativa depositada en la educación, pues ésta debía preparar al ciudadano para su adaptación y desenvolvimiento 
en una sociedad científico-tecnológica y democrática en constante cambio (ideas que recuerdan a las de John Dewey). Por entonces era una tendencia regional el depositar amplias expectativas en la educación, tanto para los individuos como para la sociedad (Stufflebeam, Kellaghan y Álvarez, 1982: 197).

Los objetivos de la educación primaria recuperados por Bolaño incluían el desarrollo de actitudes epistémicas basadas en la observación y la experiencia, así como conceptos y principios de las ciencias naturales y sociales que debían servirle al niño para explorar sus intereses y habilitarlo para aprendizajes ulteriores. Los valores científicos tenían un papel importante en la visión global de lo que debía ser la educación primaria, pues dos de los cinco criterios emanados del artículo $3^{\circ}$ constitucional que debían seguirse en los planes y programas tenían que ver con ello:

\begin{abstract}
ACTITUD CIENTÍFICA: Consiste en desarrollar en los escolares, la actitud de interrogación, de examen, de revisión, de formulación de juicios previa verificación; de combatir el dogmatismo, entendido como una actitud cerrada a la crítica. La actitud científica impregna los programas como una función orientadora y un propósito de las prácticas y actividades de maestros y alumnos.

RELATIVIDAD: Como resultado del desarrollo de la actitud científica y la adopción de la conciencia histórica, se llega a la convicción de que los conocimientos se revisan y modifican constantemente. (SEP, 1972c: 9)
\end{abstract}

Así, los Comentarios daban un lugar prominente a la ciencia, pero con significados diversos, incluyendo los tres principales objetivos de la educación en ciencia que se han alternado y resignificado a lo largo de su historia internacionalmente: 1. La ciencia como conocimiento valioso en sí mismo, 2. La ciencia para el desarrollo personal y social, 3. Los métodos de la ciencia como medio para distintos fines (Bybee y DeBoer: 1994). Dos significados adicionales que aparecieron en menor medida en los Comentarios -y que aparecerían también en los libros de ciencias naturales de primaria- fueron la ciencia como opuesta a los fanatismos, los prejuicios y la ignorancia (SEP, 1972c: 8-9) y la ciencia como riesgo, pues el documento termina con la advertencia de que, si se valoran solo las producciones materiales, en olvido de los valores humanos y sociales, se corre el peligro de convertir a la ciencia y a la técnica "en mecanismos inconscientes de la maquinaria de producción, del odio o la destrucción". Este riesgo, como señala el mismo texto, se había corrido sentidamente en décadas anteriores con la amenaza nuclear (SEP, 1972c: 14). (El tema de la ciencia como riesgo cobraría nuevos bríos más adelante, durante la segunda revolución).

Más allá de las ideas sobre la ciencia y la educación que estaban en el ambiente, ¿cuáles fueron algunos puntos de partida y fuentes para la elaboración del programa y los libros de texto? En su libro de ensayos, Gutiérrez-Vázquez (2004) narró sus propias experiencias como 
estudiante, profesor, científico y gestor educativo a partir de las cuales forjó ideas sobre por qué y cómo enseñar y aprender ciencias. Por ejemplo, daba gran importancia a la experimentación y la observación empíricas, desde sus acercamientos al laboratorio en la infancia hasta su trabajo como formador de microbiólogos en el Politécnico. También daba gran valor a la difusión del conocimiento científico en un país como México.

Gutiérrez-Vázquez refiere que, al momento de elaborar la propuesta curricular, el equipo que encabezó no contaba con los insumos especializados que aparecerían más adelante, y que ellos mismos producirían en años posteriores. Estimó que el proyecto curricular estuvo influenciado por la corriente del 'aprendizaje por descubrimiento'. Eventualmente reconoció las críticas a ese enfoque y dio cabida a nuevas ideas; especialmente las de la psicología genética, al incorporarla como línea de investigación en el DIE-CINVESTAV y como base para un replanteamiento curricular. Pero en general, los escritos de Gutiérrez-Vázquez no parecen centrarse en adscripciones a corrientes o teorías. Esto parece congruente con la libertad que infundió en su equipo para incluir, discutir y desarrollar contenidos a partir de lo que creyeran relevante, y no a partir de compromisos teóricos.

Antonia Candela (comunicación personal, noviembre 2015) refiere que el resto del equipo, a su vez, tampoco partía de compromisos teóricos sobre pedagogía o epistemología, sino de sus propias concepciones sobre la ciencia provenientes de su formación científica, de sus posiciones políticas y de sus visiones culturales, por lo que incluso caracterizó sus ideas como "ingenuas y espontáneas". Revisaron materiales didácticos de otros países, tales como With Objectives in Mind del Reino Unido, o los de la American Association for the Advancement of Science; pero fueron las experiencias docentes de varias y varios de ellos en escuelas activas mexicanas (como la Bartolomé de Cossío) las que más les influyeron didácticamente. Otras ideas vinieron de la pedagogía de Freinet, pues también se integraron docentes de Iztapalapa, donde algunas escuelas practicaban ese enfoque. Asimismo, el equipo ponía en juego sus reflexiones conjuntas sobre el funcionamiento de las escuelas mexicanas y sobre las características y el pensamiento de las niñas y niños. Uno de los pocos compromisos teóricos se manifestó en la resistencia que opusieron ante ciertos lineamientos de corte conductista que la SEP quería imponer. Las reflexiones conjuntas les Ilevaron a proponerse innovar mediante la confección de libros que condujeran a los maestros y maestras a llevar a la práctica la conocida máxima de poner al estudiante en el centro y volverlo un sujeto activo del aprendizaje (Gutiérrez-Vázquez, 1995: 33). Se propusieron también actualizar la información científica y vincularla con las características y necesidades de la sociedad del momento. Así, aunque fue un equipo donde lideraron y predominaron las y los científicos, sus puntos de partida e intereses no se limitaban a sus propias disciplinas, sino que veían en su encargo una misión social y cultural de gran calado. 


\section{Los libros de texto, principal vehículo de la reforma}

Durante los primeros cuatro años de la reforma curricular se produjo la primera generación de libros de texto de todos los grados, con sus respectivos Auxiliares Didácticos para docentes (Ilamados 'Libros para el Maestro' en años posteriores). Comenzaron a usarse en 1972 y tuvieron una gran longevidad, tomando en cuenta los estándares mexicanos actuales: los de $1^{\circ}$ a $3^{\circ}$ tuvieron una vigencia de nueve años, mientras que los de $4^{\circ}$ a $6^{\circ}$ duraron alrededor de veinticinco, hasta ser sustituidos en 1997 por los de la reforma de 1993 (Candela et al., 2012: 15).

Los libros de texto fueron el único material por el cual la reforma curricular fue introducida y conocida, tanto por la opinión pública, como por docentes y estudiantes, ya que no se socializaron otros documentos como planes y programas (aunque existieron), y no hubo una capacitación sustantiva a docentes en servicio (fue en una sola sesión y "en cascada") ni en las Normales (no se actualizaron sus planes de acuerdo al nuevo currículo) (Gutiérrez-Vázquez, 1995: 36-37). Para Candela, esta introducción vertical del currículo no favoreció la comprensión del nuevo enfoque por parte del magisterio, y generó resistencia ante los cambios que exigía. Además, el hecho de que "universitarios" ajenos al normalismo hubieran elaborado los libros, generó una fuerte movilización de resistencia sindical y normalista (comunicación personal, noviembre 2015). Para muchas maestras y maestros que sí se dispusieron a implementar la reforma, representó un desconcierto respecto de su práctica habitual. Por ejemplo, una maestra narra que se entusiasmó con el nuevo enfoque y trató de poner en práctica los experimentos de los libros, pero esto generó desorden y pláticas entre estudiantes a las que no estaba acostumbrada; además, sus observaciones inesperadas y las preguntas que no podía responderles la hacían sentir muy mal (Dávila y Valadez, 1982: 189-190).

El equipo del DIE-CINVESTAV se dio cuenta de que la estrategia prescriptiva de arriba hacia abajo no funcionaba, y a su rol de desarrollo curricular añadió los de piloteo, investigación cualitativa y formación docente. Esto derivó en eventuales cambios al mismo currículo, así como en el desarrollo de otros programas encargados por la SEP, tales como Dialogar y Descubrir del Consejo Nacional de Fomento Educativo (CONAFE), Primaria Intensiva 11-14, y el Plan de Actividades Culturales de Apoyo a la Educación Primaria (PACAEP) (GutiérrezVázquez, 1995; Gutiérrez-Vázquez et al., 1993; De Ibarrola, 1989).

Aquí nos centraremos en analizar los libros en sí mismos, pues fueron el principal vehículo de la reforma, al menos en los primeros años. En ellos, el equipo reformador plasmó sus diversas visiones sobre la ciencia, su enseñanza y su aprendizaje, y su significado en la sociedad mexicana.

Empezaremos con aspectos que revelan la influencia de autores como Jerome Bruner, así como de visiones más tradicionales de la ciencia, y pedagogías basadas en que las y los 
niños sean partícipes de la ciencia "tal como es" (aunque veremos que en esto también hubo importantes matices). Después analizaremos aspectos que dan el contrapunto a lo "positivista" de la reforma: la presentación de las disciplinas científicas con sus vanguardias y controversias, las dimensiones sociales de las ciencias y de los fenómenos naturales, las perspectivas de izquierda y de "tercermundismo", la atención a los riesgos sociales y ambientales de la ciencia y la tecnología, y el énfasis en lo rural, lo campesino y lo popular en el currículo.

Comencemos con un vistazo al temario de los libros. Las primeras seis unidades de primer grado invitan al ejercicio de los cinco sentidos, y la percepción directa se presenta como primer paso del proceder científico. Después, a lo largo de los seis grados, es patente el peso de todo lo relativo a los seres vivos, y especialmente, al campo. También aparecen temas de otras disciplinas como física, química y astronomía. La mayoría de las unidades tienen nombre de conceptos ("Los seres vivos", "El calor"), o bien, pequeñas proposiciones sobre fenómenos naturales ("El viento modifica el terreno", "Todas las cosas están hechas de partículas muy pequeñas"). Otros más son interpelaciones a los niños y niñas sobre su entorno o sus hábitos ("El mundo en que vives", "Cómo cuidar tu cuerpo"). Temas similares se van repitiendo a lo largo de los grados, pero cada vez con mayor sofisticación conceptual, mayor alejamiento del sentido común, y un lenguaje más especializado. La progresión y la reiteración de los temas a lo largo de la primaria revela la influencia de Bruner y su currículo en espiral. El contenido y su ordenamiento contrasta con la generación anterior de libros de texto, en los que predominaban las recomendaciones de higiene y nutrición en los primeros grados, y los contenidos más disciplinares en los últimos grados.

La estructura de los Auxiliares Didácticos para docentes revela otra lógica subyacente al currículo: en cada lección se especifica cuáles de las cinco "ideas generales" se abordarán: unidad, diversidad, interacción, cambio y ciencia como fenómeno social. Se explica que, "En lugar de paquetes de información, lo que importa es saber manejar unas cuantas ideas generales que den estructura a todas las Ciencias Naturales" ( $1^{\circ}$, A.D.: 6$)^{2}$, (idea que ya estaba presente en los libros de Gerald Spellman Craig en los años 1920s). Los Auxiliares Didácticos también especifican los objetivos de aprendizaje de cada lección, formulados en términos de "procesos de indagación científica". Por ejemplo, en la unidad "El clima y el tiempo" de $6^{\circ}$ grado, estos objetivos incluían "Comprobar experimentalmente la fuerza que ejerce la presión atmosférica sobre los cuerpos. ... Comprobar experimentalmente la formación de corrientes de aire. Medir algunas características del clima con aparatos construidos durante el desarrollo de la lección. Registrar sistemáticamente, mediante textos libres y

\footnotetext{
2 Dado que los datos de publicación de todos los libros son muy parecidos, las referencias los identificarán por el grado, seguido de "L.A." o "A.D.", según se trate del Libro para el alumno o el Auxiliar didáctico. Los datos completos de todos los libros están en la lista de referencias.
} 
dibujos, las observaciones y conclusiones obtenidas en la lección" (SEP, 6ª A.D.: 61). En consonancia, es frecuente la indicación de realizar distintos experimentos y actividades con los niños y niñas. Un ejemplo representativo es el experimento "para averiguar si las plantas sanas necesitan la luz" (2, A.D.: 84-86). Se presentan los pasos de la actividad, así como la referencia teórica de lo que es un experimento con hipótesis, condiciones controladas, grupo piloto, grupo testigo, etcétera. Se pretende que las niñas y niños practiquen esta forma de realizar experimentos, y lleguen por su cuenta a la conclusión de que las plantas necesitan luz para vivir. Otros experimentos eran mucho más sofisticados. Se esperaba que las y los maestros guiaran su realización, así como el diálogo para que los niños y niñas llegaran a conclusiones tales como que el sonido necesita un medio que lo transmita, o que la respiración es una combustión. (La posterior investigación en aula obligó a recalibrar ese tipo de expectativas sobre docentes y estudiantes).

Además de los experimentos, las pedagogías frecuentemente recomendadas son las del diálogo abierto, la reflexión y la elaboración de textos libres (o dibujos, en el caso de los primeros grados): se insistía en que el objetivo no era la memorización. No aparecen actividades como contestar cuestionarios o completar frases, que eran muy comunes en la anterior generación de libros de texto. En los libros para estudiantes predominan las ilustraciones y las fotografías, muchas de ellas, a modo de instructivo visual, para las actividades y los experimentos sugeridos. Los textos son breves, aunque se vuelven más largos y predominantes que las imágenes en $6^{\circ}$ grado.

En ocasiones, la epistemología y la metodología de las ciencias constituyen el tema mismo de las lecciones. Por ejemplo, uno de los textos para $5^{\circ}$ grado explica a las y los estudiantes que durante todo el año aprenderán ciencia haciendo ciencia; haciendo investigaciones como las hacen los científicos, y observando los fenómenos que la ciencia estudia en su vida diaria dentro y fuera del salón. Les dice que su maestro los dividirá en equipos, en los que harán juntos todas las investigaciones y discutirán sus etapas, sus resultados y su utilidad para mejorar el mundo (5, L.A.: 11). Las "cajas negras", presentes en diferentes grados, son otra forma en que se aborda explícitamente la metodología y epistemología de la ciencia. En esas lecciones, las niñas y niños tratan de averiguar mediante el tacto o el movimiento qué objetos están en el interior de diferentes cajas. Luego, las lecciones sugieren comparar esto con la posibilidad de estudiar incluso aquello que no puede verse, mediante modelos. En $4^{\circ}$ grado, la actividad de la caja negra se usa también para introducir los pasos del método científico, y de las investigaciones en general: observar, registrar, explicar, consultar, experimentar, distinguir y enunciar. Además de los mensajes más tradicionales sobre la ciencia "tal cual es" -el método científico y el rol de la experiencia y la experimentación-, a lo largo de los libros aparecen otros matices y posturas epistemológicas. Por ejemplo, en la unidad "Cómo resolvemos problemas" en $6^{\circ}$ grado contiene un relato sobre un campesino y unos 
niños que quieren cultivar ciruelas, y reflexionan sobre la utilidad de una formación científica en la vida cotidiana. El relato tiene alusiones eclécticas: empiristas, cartesianas, kuhnianas, popperianas y pragmatistas (6, L.A.: 16).

Parte del encargo al equipo reformador había sido presentar la realidad y la actualidad de las disciplinas científicas. Este cometido lo cumplieron al hablar sobre los objetos de estudio, las preguntas, las prácticas, las vanguardias y hasta las controversias de diversas disciplinas; sobre todo a través de información dirigida a docentes. En la unidad "Comportamiento animal" de $6^{\circ}$ grado, por ejemplo, se les informa que la etología es la disciplina que los estudia: "se ocupa tanto de los aspectos más notables o llamativos de la conducta animal como de muchos detalles que parecen insignificantes pero que tienen gran valor científico" (6 $6^{\circ}$ A.D.: 43). Luego se sopesan las ventajas relativas de los diferentes métodos de la etología, su historia, sus yerros y algunos de sus hallazgos. Numerosos detalles denotan la actualidad científica que se buscó imprimir al contenido. Por ejemplo, una unidad sobre la luna habla sobre los viajes espaciales que estaban por venir ( $1^{\circ}$, A.D.: 181); otra sobre la galaxia y el universo habla de los "poderosos telescopios" e "ingeniosas observaciones" que hacía menos de 50 años habían revolucionado la comprensión del universo, aunque sus límites y estructura general, dicen, seguían incógnitos (6 $6^{\circ}$ L.A.: 44; 6 A.D., 57-58).

Presentar las dimensiones sociales de la ciencia fue otro rasgo definitorio de estos libros, y ello ocurrió "mucho antes de que adquirieran importancia internacional las corrientes de "ciencia y sociedad", como señala Candela (1989: 10). Sobre todo, los libros presentaron las implicaciones sociales de los fenómenos naturales que estudiaban. Por ejemplo, en la unidad sobre el ciclo del agua en $4^{\circ}$ grado, el Auxiliar didáctico explica el ciclo hidrológico y sus características en México, pero también habla de la desigual distribución del agua y su contaminación en el territorio nacional (4 , A.D.: 50-51). Otras veces se aborda la sociología de la ciencia y la tecnología, con énfasis en las desigualdades entre países. Así sucede en la lección sobre viajes espaciales en $4^{\circ}$ grado: "Muchos países no pueden aprovechar los beneficios de la investigación espacial porque es muy costosa" (4, L.A.: 170). Una lección sobre el mar y sus recursos, de $5^{\circ}$ grado, habla de las dificultades para explorar el fondo del mar, y cuenta que hasta hacía poco, éste había sido un misterio completo, y solo las naciones poderosas habían dominado el océano (5, L.M.: 187).

En retrospectiva, quizá lo más llamativo sean los temas y posturas políticas presentes en estos libros oficiales de ciencias naturales. Estas posturas, según el testimonio de los y las reformadoras, fueron las suyas propias, pues contaron con total autonomía respecto del gobierno; pero el hecho es que coincidían con el discurso de izquierda y tercermundismo del sexenio de Echeverría. Como ejemplo, a propósito de la contaminación, el Auxiliar didáctico de $6^{\circ}$ grado dice que la aplicación de la ley ambiental "ha topado con la resistencia de muchos industriales que no quieren hacer las inversiones necesarias para reducir las descargas de 
contaminantes de sus fábricas", y que hay industrias "como la petrolera, la azucarera, la del transporte o la textil, que son importantes fuentes de contaminación pero que por su poderío económico representan un obstáculo a la aplicación de la ley" (6, A.D.: 117). La lección sobre el movimiento en $4^{\circ}$ grado pide a los niños empujar una mesa en distintas direcciones para mostrar el efecto de fuerzas distintas sobre los cuerpos, y luego plantea la reflexión de que, tanto en la naturaleza como entre las personas, el trabajo necesita ser organizado para llevar a un cambio, como en el caso de los obreros y los campesinos, que "juntando sus tierras y organizándose para trabajarlas colectivamente pueden enfrentar mejor los gastos y sacar más provecho de ellas. Así también aumentan su fuerza para defenderse de los terratenientes, los caciques y los intermediarios" (4 , L.A.: 83).

La dimensión social también está presente en referencias a aplicaciones cotidianas de la ciencia y la tecnología. Por ejemplo, el libro del alumno de $5^{\circ}$ le pide reflexionar sobre las revolucionarias repercusiones de la máquina de vapor en la sociedad a partir de una actividad en la que mueven un rehilete con vapor (5, L.A.: 159-160). La unidad que cierra el currículo en $6^{\circ}$ grado se titula, precisamente, "Ciencia y sociedad", y propone a las y los estudiantes imaginar su vida sin los productos y servicios de la industria y la técnica modernas, para así reconocer su impacto. El texto continúa señalando los vertiginosos adelantos y descubrimientos de los últimos 50 años, y el libro concluye con una nota de tecnoentusiasmo (la creencia de que la ciencia y la tecnología podrán resolver los grandes problemas de la humanidad, incluyendo los ocasionados por la misma ciencia y tecnología), que contrasta con el énfasis en las consecuencias negativas y los riesgos sociales y ambientales de la ciencia y la tecnología presente a lo largo de los libros:

Como puedes ver, la ciencia ha sido una de las fuerzas más poderosas en la historia de la humanidad. ... ha originado cambios en la manera de ser de la gente, en su manera de pensar, en su modo de actuar. Muchos de nuestros problemas actuales, como la contaminación ambiental, se deben a inadecuadas aplicaciones de la ciencia; pero estos problemas también se resolverán sólo con la ayuda de la ciencia misma. (6 , L.A.: 207)

En una edición posterior, esta unidad final sobre ciencia y sociedad incluye un análisis marxista de la producción de telas en la industrialización: explica cómo los pequeños talleres fueron remplazados por las fábricas a vapor puestas por comerciantes ricos, y concluye que:

En la producción fabril capitalista, las innovaciones benefician al dueño ... mientras que los trabajadores siguen recibiendo el mismo salario. Los que inventan mejores formas de hacer las cosas, son técnicos y científicos contratados por los dueños de las fábricas. (SEP, 1976: 235) 
La investigación científica, concluye esta versión del libro, está en manos de países ricos que la utilizan para mantener su poderío, dejando sin realizar su potencial para mejorar la condición humana.

Una última manera en que se refleja la vocación social de estos materiales es la fuerte presencia de lo rural, lo campesino y lo popular, tanto en los contenidos como en la estética. Las y los autores eran conscientes de que estas realidades coexistían con la del México más moderno, industrial y urbanizado, e hicieron un evidente esfuerzo por que todas las niñas y niños se vieran reflejados. Además, la didáctica sugerida implicaba muchas salidas al exterior, al campo, y el contacto y trabajo con plantas y animales. Incluso se explica a los padres de familia que "Para observar e investigar, con frecuencia será necesario que los niños salgan del salón de clase, ya sea al patio de la escuela, al jardín más cercano o al campo" (2, L.A.: 3). El trabajo de campo se pauta detalladamente. Por ejemplo, se indica la recolección y clasificación de ejemplares, o la conformación de un 'rincón vivo' dentro del aula. En algunas ocasiones solo se sugiere sacar a los niños al campo, sin especificar cómo aprovechar la salida para la lección por ejemplo, en la unidad "Vivimos en la superficie de un astro: La Tierra", ( $1^{\circ}$, A.D.: 68). Muchas lecciones implican un verdadero saber agrícola, tanto teórico como práctico, por parte de docentes y estudiantes. En $4^{\circ}$ grado, por ejemplo, se pretende que lleven a la práctica aprendizajes sobre la erosión, los fertilizantes y la rotación de cultivos (4 , A.D.: 101). Varias lecciones insisten sobre los perjuicios de la erosión y el deber de cuidar el suelo para el cultivo: "Tenemos que procurar no dejar terrenos sin plantas..." (5, L.A.: 188).

A pesar del lugar preponderante de la agricultura y de las experiencias con el campo, las plantas y los animales; nunca se sugiere solicitar el saber de las familias u otras personas en tanto campesinas o indígenas. El entorno natural a veces aparece como una fuente de conocimiento por el contacto empírico con él, pero no por los saberes de las comunidades del campo. Cuando sí se habla de la experticia del campo, se la atribuye a los "hombres de ciencia"; no a los campesinos. Cuando las actividades requieren orientaciones prácticas, se sugiere escribir a "las dependencias oficiales encargadas de los asuntos relacionados con la agricultura, solicitando información o asesoramiento sobre el cuidado del suelo y su aprovechamiento óptimo" ( $2^{\circ}$, A.D.: 128) y "recurrir a los servicios de extensión agrícola de las agencias de la Secretaría de Agricultura y Ganadería" (5, A.D.: 176), o se remite a los "laboratorios especializados que tiene el gobierno" para saber qué fertilizantes le faltan a un suelo ( $3^{\circ}$, A.D.: 65). Se habla de los adelantos tecnológicos que multiplican la productividad del campo, como los granos de alto rendimiento, y se lamenta que "en México y los demás países latinoamericanos predomina todavía una agricultura atrasada a base de técnicas rudimentarias de bajo rendimiento" (6 $6^{\circ}$ A.D.: 91). Irónicamente, fue en muchas de las escuelas rurales donde las investigadoras del DIE-CINVESTAV pudieron constatar que los y las 
docentes sí realizaban las actividades agrícolas que el currículo sugería (Candela, A. Comunicación personal, octubre de 2015).

En conjunto, los libros ofrecían una visión sobre los fenómenos naturales, sobre las ciencias, la tecnología y la sociedad; todo ello desde perspectivas epistemológicas, políticas y estéticas definidas. Buscaban también transmitir una visión sobre la forma en que las niñas y niños desarrollarían los aprendizajes esperados mediante su interacción con objetos, plantas y animales, en diálogo y colaboración con las y los demás.

\section{Conclusiones}

En este artículo se analizaron los antecedentes, el contexto y el proceso por el cual se encargó y se diseñó el currículo de ciencias naturales de primaria en los setenta. Se analizaron también los libros de texto, que fueron el principal vehículo de la reforma, y que se convirtieron en el material que tantos millones en México tuvieron durante décadas.

A veces se ha explicado esta reforma desde la investigación educativa como una réplica con retraso de la "primera revolución" estadounidense en la enseñanza de las ciencias, que promovía una enseñanza "dura" de las disciplinas científicas mediante el método científico y la experimentación. Aquí se ha analizado con una mirada histórica, filosófica y social desde el Sur, que busca ir más allá de esta explicación, restituyendo un significado propio de este episodio para la historia de la educación y la historia social de la ciencia.

A pesar de lo que los términos "reforma" y "revolución" pudieran sugerir, este proyecto de renovación curricular en realidad abrevaba de largas tradiciones del mundo occidental. Por un lado, retomaba tradiciones de educación en ciencia, misma que había llegado para quedarse a la educación básica de los modernos estados nación desde el siglo XIX. Retomaba también elementos de la pedagogía progresista europea, que formaba parte del pensamiento educativo mexicano desde hacía más de un siglo (y continúa haciéndolo). Abrevaba, finalmente de la cultura científica de la que las y los reformadores eran partícipes, y de sus visiones sobre aquello que la formación en ciencia debía aportar a la niñez y a la sociedad mexicana. La consonancia con la primera revolución y sus herencias (evidente en la organización conceptual y en espiral del currículo, así como en el énfasis empírico y experimental y la información disciplinar), conviven en los libros de texto con las visiones de los autores y autoras en torno a los fenómenos naturales, el campo, la ciencia y la tecnología en la sociedad mexicana de la época.

El régimen de Echeverría brinda otras tantas claves para interpretar históricamente esta reforma. Por un lado, la ambiciosa política educativa y científico-tecnológica del sexenio (que en parte respondía a la necesidad del régimen priísta de legitimarse tras su desprestigio por la matanza de Tlatelolco), enmarcó la decisión de renovar el currículo, y de confiarlo por 
primera vez a científicos y científicas de instituciones de educación superior, incluyendo a disidentes del sexenio anterior. Esta política educativa también auspició que el grupo reformador no solo diseñara el currículo, sino que conformara el primer programa mexicano de investigación y desarrollo en educación en ciencia en el DIE-CINVESTAV. Finalmente, el régimen echeverrista brinda el marco ideológico y retórico -de izquierda, tercermundista y popular- que, en conjunción con las visiones del equipo reformador es patente en los libros de texto.

En México como en otros lugares, las reformas educativas han sido instrumentos de los gobiernos para comunicar legitimidad, renovación y avance; para dejar su impronta y para difundir sus ideas. Se han implementado de arriba abajo, de forma prescriptiva y en cascada. Aunque se sabe bien que estas prácticas no funcionan, persisten. Esto es algo que el equipo reformador de los setenta después reconoció y buscó remediar. Por lo mismo, un panorama completo de lo que significó la ciencia en la educación tendría que tomar en cuenta lo que realmente se vivió en las escuelas a raíz de, y a pesar de, la reforma curricular (el mismo DIE-CINVESTAV realizó investigaciones de campo al respecto), así como otras formas de educación científica, tecnológica, agrícola y ambiental que probablemente no pasaron por el sistema educativo oficial. Con todo, esta reforma, como todas, fue producto de un proceso complejo. Revisar sus ricos antecedentes, su contexto y su contenido -más allá de su ubicación entre "revoluciones"- abre ventanas para la historia de la educación y la historia social de la ciencia y la tecnología en México. 


\section{Referencias hemerográficas}

Candela, María Antonia (1989), "Los libros de texto gratuitos de ciencias naturales y la investigación en la enseñanza de las ciencias" en Avance y Perspectiva. Órgano de Difusión del CINVESTAV-IPN, Ciudad de México, 37, pp. 5-13.

Dávila, M. y Valadez, M. (1982), "Testimonios. Dos versiones de un mismo quehacer" en Educación: Revista del Consejo Nacional Técnico de la Educación, vol. 8, núm. 42, pp. 187-200.

De Ibarrola, María (1989), "La evaluación del trabajo académico en investigaciones educativas" en Avance y Perspectiva. Órgano de Difusión del CINVESTAV-IPN, Ciudad de México, 37, pp. 55-62.

Duschl, Richard (1985), "Science Education and Philosophy of Science. Twenty-Five Years of Mutually Exclusive Development" in School Science and Mathematics, vol. 85, núm. 7, pp. 541-555.

Gutiérrez-Vázquez, Juan Manuel (1984), "Tendencias más importantes en la enseñanza contemporánea de la ciencia" en Revista de la Educación Superior, Asociación Nacional de Universidades e Instituciones de Educación Superior, Ciudad de México, núm. 4 (52), vol. 13, pp. 43-52.

-(1995), "La reforma de 1972-1976" en Básica. Revista de la Escuela y del Maestro, Sindicato Nacional de Trabajadores de la Educación, Ciudad de México, núm. 4, pp. 30-38.

María Victoria Avilés, Rubén Montañez y Abelardo Mejía (1993), "Libros de texto y estilos de docencia. Uso de los libros de ciencias naturales en el Estado de Michoacán" en Revista Latinoamericana de Estudios Educativos, Centro de Estudios Educativos, Ciudad de México, vol. 23, núm. 4, pp. 87-107.

\section{Referencias bibliográficas}

Akker, Van (1998), "The science curriculum: between ideals and outcomes", en Barry Fraser y Kenneth Tobin (eds.), International Handbook of Science Education, I, Kluwer Academic Press, Países Bajos, pp. 421-447.

Azuela, Luz Fernanda (2010), "La ciencia positivista en el siglo XIX mexicano" en Rosalba Ruiz, Arturo Argueta y Graciela Zamudio (coords.), Otras armas para la Independencia y la Revolución, Fondo de Cultura Económica, México, pp. 172-188.

Bybee, Rodger y George E. DeBoer (1994), "Research on goals for the science curriculum", en Dorothy Gabel (ed.), Handbook of Research on Science Teaching and Learning, Macmillan, Nueva York, pp. 357-387.

Bruner, Jerome (1960), The process of education, Harvard University Press, Cambridge.

Candela, María Antonia, Armando Sánchez y Clara Alvarado (2012), "Las ciencias naturales en las reformas curriculares", en Fernando Flores (coord.), La enseñanza de la ciencia en la educación básica en México, Instituto Nacional para la Evaluación de la Educación, México, pp. 11-32.

Fuentes, Olac (1979), "Educación pública y sociedad" en Pablo González-Casanova y Enrique Florescano (coords.), México hoy: Entre lo deseable y lo posible, Siglo XXI, México, pp. 230-265.

Gorbach, Frida y Carlos López (2008), "Apuntes para ubicar nuestras historias de las ciencias", en Frida Gorbach y Carlos López (eds.), Saberes locales. Ensayos sobre historia de la ciencia en América Latina, El Colegio de Michoacán, México, pp. 11-38.

Gutiérrez-Vázquez, Juan Manuel (2004), Con paso lento y agitadamente. Ensayos educativos, DIECINVESTAV, México.

Latapí, Pablo (1998), "Un siglo de educación nacional: una sistematización", en Pablo Latapí (coord.), Un siglo de educación en México, Fondo de Cultura Económica, México, pp. 21-42. 
Leff, Enrique (1979), "Dependencia científico-tecnológica y desarrollo económico", en Pablo GonzálezCasanova y Enrique Florescano (coords.), México Hoy: Entre lo deseable y lo posible, Siglo XXI, México, pp. 266-285.

León, Ana Isabel (et al) (1995), "Ciencias Naturales y Tecnología”, en Guillermina Waldegg (ed.), Procesos de Enseñanza y Aprendizaje II, Consejo Mexicano de Investigación Educativa, México, pp. 21-120.

López, Ángel, D. y León, Ana Isabel (2003), "Currículo como estructura y proceso", en Ángel López (coord.), Saberes científicos, humanísticos y tecnológicos: procesos de enseñanza y aprendizaje (Tomo I), Consejo Mexicano de Investigación Educativa, México, pp. 369-397.

McCormack, Alan (1992), "Trends and Issues in Science Curriculum", en Denis W. Cheek (ed.), Science Curriculum Resource Handbook: A Practical Guide for K-12 Science Curriculum, Kraus International Publications, Nueva York, pp. 16-41.

McNeill, John Robert y William McNeill (2003), The human web: a bird's-eye view of world history, WW Norton \& Company, Nueva York.

Meneses, Ernesto (1991), Tendencias educativas oficiales en México, 1964-1976, Centro de Estudios Educativos/Universidad Iberoamericana, México.

Núñez, María Salud (1980), Diagnóstico de la enseñanza de las Ciencias Naturales en la escuela primaria, Informe de Investigación. Documento interno, DIE-CINVESTAV-IPN, México.

Secretaría de Educación Pública (1972a), Aportaciones al estudio de los problemas de la educación, SEP, México.

- (1972b), Ciencias Naturales. Auxiliar didáctico para el primer grado, SEP, México.

- (1972c). Primera Asamblea Nacional de Educación Primaria. Comentarios a los fundamentos del plan de estudios y programas de educación primaria, SEP, México.

------- (1972d). Ciencias Naturales. Auxiliar didáctico para el segundo grado, SEP, México.

(1972e). Ciencias Naturales. Auxiliar didáctico para el tercer grado, SEP, México.

- (1972f). Ciencias Naturales. Primer grado, SEP, México.

(1972g). Ciencias Naturales. Segundo grado, SEP, México.

(1973a). Ciencias Naturales. Quinto grado, SEP, México.

(1973b). Ciencias Naturales. Quinto grado. Auxiliar didáctico para el quinto grado, SEP, México.

(1973c). Ciencias Naturales. Tercer grado, SEP, México.

(1974a). Ciencias Naturales. Cuarto grado. Libro del maestro, SEP, México.

(1974b). Ciencias Naturales. Sexto grado, SEP, México.

(1974c). Ciencias Naturales. Sexto grado. Libro del maestro, SEP, México.

(1977). Ciencias Naturales. Cuarto grado, SEP, México.

Stufflebeam, Daniel; Kellaghan, Thomas y Álvarez, Benjamín (1982), La evaluación educativa. Evidencias científicas y cuestionamientos políticos en torno a la evaluación educativa, Pontificia Universidad Javeriana, Bogotá.

Wallace, John y Louden, William (1998), "Curriculum changes in science: riding the waves of reform", en Barry Fraser y Kenneth Tobin (eds.), International Handbook of Science Education, I, Kluwer Academic Press, Países Bajos, pp. 471-485. 


\section{Referencias electrónicas}

DIE-CINVESTAV. [Canal de YouTube de profesores del DIE-CINVESTAV] (2014). Historias y prospectivas académicas. EI DIE en el 50 aniversario del CINVESTAV. Evento inaugural: A 40 años de su creación. Homenaje a Juan Manuel Gutiérrez-Vázquez. Disponible en https://www.youtube.com/ watch?v=ceVQFLavaD8

Pedretti, Erminia y Nazir, Joanne (2011), "Currents in STSE Education: Mapping a Complex Field, 40 Years On" in Science Education, vol. 95, núm. 4, pp. 601-626. https://doi.org/10.1002/sce.20435 